\title{
Phorate Induced Hepatotoxicity in Rats
}

\author{
sangeetha G S, muraleedhara Kurup, helen A \\ Dept. of Biochemistry, University Of Kerala, Thiruvananthapuram
}

\begin{abstract}
Organophosphorus compounds induce oxidative stress leading to generation of free radicals and alterations in antioxidant and scavengers of oxygen free radicals. The present study demonstrates the effect of chronic exposure of phorate in causation of oxidative stress in male Sprague-Dawley rats. Phorate was administered orally at doses $0.05,0.1$ and $1 \mathrm{mg} / \mathrm{kg}$ of body weight for 60 days. After the administration of doses, the liver and blood samples were analyzed for various parameters of oxidative stress and liver toxicity markers. The results indicated an increase in lipid peroxidation, increase in the levels of aminotransferases (ALT and AST), ALP,ACP, LDH and GGT .Similarly, there was a dose dependent decrease in catalase, superoxide dismutase and GSH in liver at all doses. The level of cytochrome P450 was also found to be enhanced. Histopathological changes confirmed that phorate caused significant structural damages to the liver tissues. These results might perhaps be the first to establish the phorate induced oxidative stress mediated hepatotoxicity in rats.
\end{abstract}

\section{Introduction}

Phorate is an Organophosphorous insecticide and acaricide used to control sucking and chewing pests in a wide range of crops, among others corn, sugar beets, cotton, brassicas, and coffee. It is also used as a nematocide (ACG99, Gal91, Tom97). It is also used in pine forests and on root and field crops, including corn, cotton, coffee, and some ornamental plants and bulbs (Abhilash and Singh, 2009; Gan and Jans, 2007). Phorate has been classified as a Class I, with an LD50 of 1.1 to $3.7 \mathrm{mg} / \mathrm{kg}$ bw for rats (http://extoxnet.orst.edu/pips/phorate.htm). The United States Environmental Protection Agency (USEPA) has authorized restrictions on the use of phorate since 1990 (Devine and Furlong, 2007). However, it is continuously being used in several countries like India Italy, China, Egypt and (Abhilash and Singh, 2009; Mansour et al., 2009; Pagliuca et al., 2006;Wang et al., 2008). OP pesticides, in addition to their intended effects like the control of insects or other pests, are sometimes found to affect non target organisms including humans (Chantelli-Forti et al., 1993; Chaudhuri et al., 1999). It has been reported that OPs may induce oxidative stress on acute exposure in humans (Banerjee et al., 1999; Almedia et al., 1997) and animals (Vandana and Poovolla, 1999; Dipanker and Tapas, 2000).

In general, OPIs are neurotoxic in nature by acting as inhibitors of neuronal cholinesterase (ChE) activity. However, some studies reported that OPIs caused lipid peroxidation (LPO). In these studies, LPO has been suggested as one of the molecular mechanisms involved in OPI-induced toxicity (Gupta et al., 1992; Yamano and Morita 1992; Bagchi et al., 1995; Bachowski et al., 1997; Gultekin et al. 2000b, 2001).The cellular antioxidant pool comprises of integral antioxidants like glutathione and other thiols, and antioxidant free radical scavenging enzymes like catalase (CAT), superoxide dismutase (SOD), glutathione (GSH). Longterm oxidative stress is also expressed as changes in extent of lipid peroxidation . The objective of this study is to evaluate the effect of phorate -induced oxidative injury in liver of rats. We have planned to study the effects of phorate in the causation of oxidative stress mediated hepatotoxicity following chronic exposure to phorate in rats and also to investigate the ensuing histopathological changes in liver and brain of these experimental animals.

\subsection{Materials}

\section{Materials and methods}

Phorate (purity 95.8\%) supplied by Merck Ltd. was used for the study. Refined groundnut oil was used for preparing suspensions of phorate. All other chemicals used were of analytical grade.

\subsection{Animals}

The dose of the pesticide used was calculated from the LD 50 given in the literature and safe dose was selected after a pilot study. Male Sprague-Dawley rats, aged 20-21 weeks and weighing $160 \pm 20 \mathrm{~g}$, were selected from the same inbred colony maintained in the animal house (under the supervision of the Animal Committee) under controlled conditions of temperature at $25 \pm 2^{\circ} \mathrm{C}$, relative humidity of $50 \pm 15 \%$ and normal photoperiod ( $12 \mathrm{~h}$ dark: $12 \mathrm{~h}$ light). The animals were given sterile food pellets and water ad libitum. Animals were housed throughout the experiment in polypropylene cages (with each cage housing six animals) and 
allowed to acclimatize to the laboratory environment for 7 days. The design of the study was in accordance with the ethical guidelines prescribed by the University.

\subsection{Experimental design}

Rats were randomly divided into four groups each containing six animals. Route of administration selected for the study was oral (using 22-gauge oral feeding needles). Group P1 animals were fed groundnut oil by oral gavage treated as control while groups P2, P3, P4 animals were fed phorate in groundnut oil at doses $0.05,0.1$ and $1 \mathrm{mg} / \mathrm{kg}$ body weight corresponding to LD50 (Gallo and Lawryk, 1991) in a similar fashion and sacrificed using light ether anaesthesia after 60 days of treatment.

\subsection{Sample collection and Preparation of tissue homogenate}

Rats were anaesthetized with light ether and venous blood samples were collected by direct heart puncture into sterilized vials. Blood samples allowed setting to clot at $4^{\circ} \mathrm{C}$ and centrifuged at $1000 \mathrm{~g}$ for 30 $\min$. Then $1000 \mu \mathrm{l}$ aliquots of serum were placed in microfuge tubes and frozen on dry ice. Labeled bags were placed into a $-20^{\circ} \mathrm{C}$ freezer until the time of the assay. Liver tissues were removed and washed with ice-cold saline. Tissues were separately homogenized for $5 \mathrm{~min}$ in four volumes of ice-cold $0.1 \mathrm{M}$ phosphate buffer, $\mathrm{pH}$ 7.4, containing $0.15 \mathrm{M} \mathrm{KCl}$. The homogenate was centrifuged at $9000 \times g$ at $4{ }^{\circ} \mathrm{C}$ for $20 \mathrm{~min}$. The supernatant was pipetted into a clean centrifuge tubes and centrifuged further at $104,000 \times g$ at $4{ }^{\circ} \mathrm{C}$ in ultracentrifuge. Microsomal and cytosolic fractions were used for the study.

\subsection{Histopathology}

Liver and brain tissues were fixed in $10 \%$ formalin, routinely processed, embedded in paraffin and sections of $5 \mu \mathrm{m}$ thick were cut.

\subsection{Biochemical assays}

Lipid peroxidation (LPO) was measured in hepatic tissue homogenates according to the method (Ohkawa et al., 1979) based on the of thiobarbituric acid reactive substances (TBARs) and expressed as the extent of MDA production. Superoxide dismutase (SOD) activity was measured in serum according to Flohe and Otting (1984). Catalase activity in serum was assayed by monitoring the decomposition of $\mathrm{H} 2 \mathrm{O} 2$ at 240 $\mathrm{nm}$ as described by (Claiborne, 1985). Serum alanine aminotranseferase (ALT) and aspartate aminotranseferase (AST) activities were determined using commercial kits obtained from Bio M'erieux, France. The principle reaction of the colorimetric determination of AST or ALT activity is based on the reaction of aspartate or alanine with $\alpha$-ketoglutarate to form oxaloacetate or pyruvate hydrazone formed with 2, 4-dinitrophenylhydrazine (Reitman and Frankel, 1957). Serum alkaline phosphatase (ALP) activity was measured at $405 \mathrm{~nm}$ by the formation of para-nitrophenol from para-nitrophenylphosphate as a substrate (King and Armstrong, 1934). Acid phosphatase activity (ACP) was determined according to Tietz (1986) using commercial kits obtained from Bio ADWIC, Egypt. Serum lactate dehydrogenase (LDH) was determined according to the method of Bergmeyer et al., 1974. The protein content was estimated by the method of Lowry et al. with bovine serum albumin as the standard. Serum gamma glutamyl transferase $(\gamma-\mathrm{GT})$ was measured according to the method of Tietz (1986), using commercial kits obtained from BioSystems Co., Spain. Cytochrome P450 was assayed in hepatic microsomes according to the method of (Omura and Sato, 1964) and its activity was expressed as nmol/mg protein.

\section{Results}

The effect of oral administration of phorate on Cytochrome P450 in liver of rats is depicted in Fig. 1. Cytochrome P450 was found to be significantly raised $(P \leq \mathrm{s} 0.05)$ in animals treated with phorate when compared to controls. As shown in Fig 2, rats fed with an oral dose of phorate developed severe liver damage as indicated by the increased serum levels of the liver damage marker enzymes ALP, ACP, AST and ALT with respect to control. Superoxide dismutase activity was recorded to be decreased in treated groups when compared to control group (Fig3). Similarly, the catalase activity was also found to be reduced in a dose dependent manner in liver as depicted in Fig.3B . Phorate treatment also induced lipid peroxidation. The results are presented in Fig.4A. Phorate significantly increased LPO in liver at all doses as compared to control values. Glutathione content was decreased by phorate in a dose dependent manner (Fig.4B).

The histopathological changes of liver of experimental animals were compared to those of controls and illustrated in Fig.5. In treated animals, pyknotic nuclei (PN), fatty infiltrations (FD), inflammatory leukocyte infiltrations (LI) and activated Kupffer cells (KC), dilatation in blood sinusoids (DBS) and cytoplasmic vacuoles $(\mathrm{VC})$ were found which indicate hepatocellular damage. 


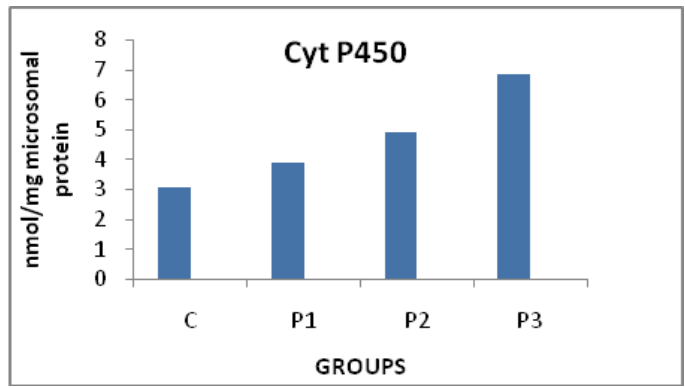

Fig. 1. Effect of phorate treatment on Cytochrome P450 of rat liver. Values are expressed as mean \pm S.E. of six animals in each group.

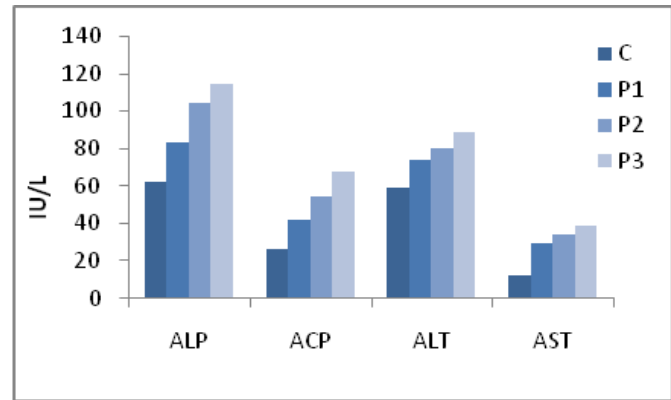

Fig.2 Liver damage following pesticide exposure as shown by marker enzymes.

Values are mean \pm S.D. from 6 rats.
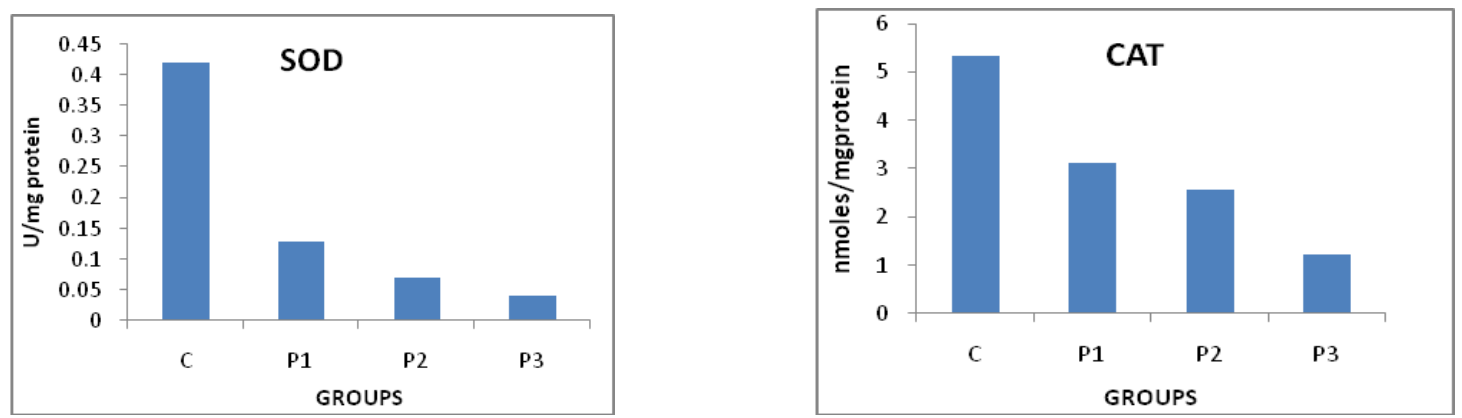

Fig. 3. Effect of phorate on liver SOD and catalase of the control and experimental rats.

Values are mean \pm S.D. from 6 rats .
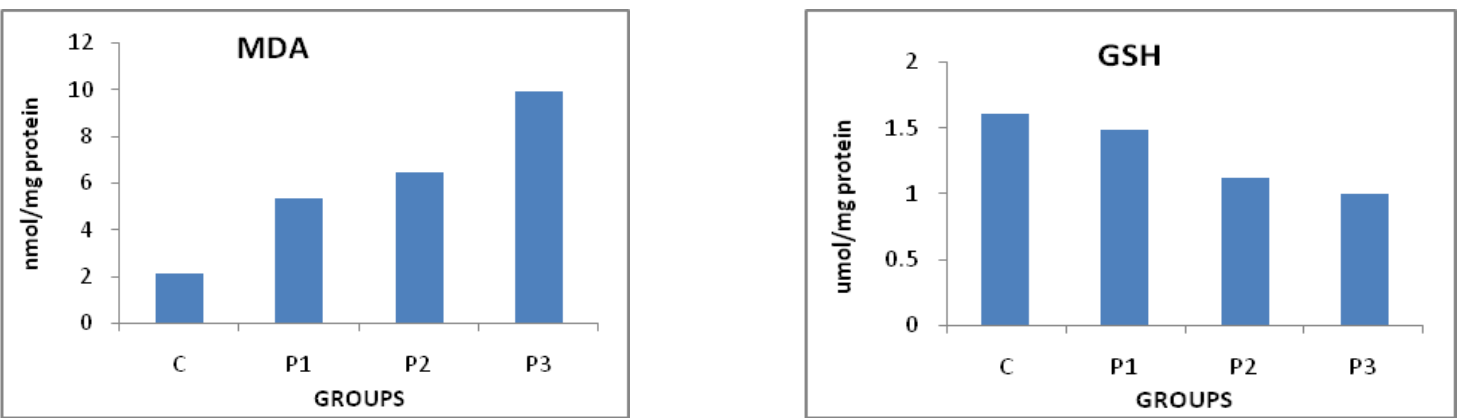

Fig. 4. Effect of phorate treatment on lipid peroxidation and GSH of the control and experimental rats. Values are mean \pm S.D. from 6 rats . 

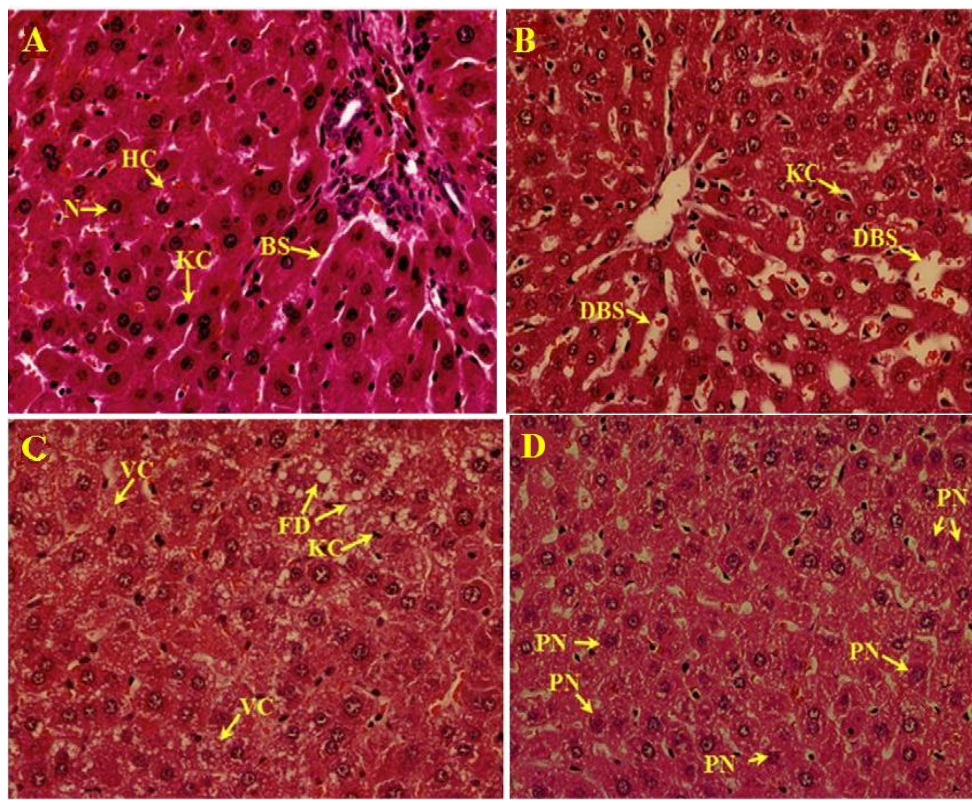

Figure 5. Photomicrograph of rat liver after consecutive daily oral administration of phorate at different doses for 60 days A: liver sections of control animals showing normal histological appearance of the liver, including central vein (CV), blood sinusoids (BS), hepatic cells (HC), Kupffer cell (KC) and centrally located nuclei (N). (H\&E, 100x) B-D: liver sections of phorate treated animals (P1-0.5, P2-1.0 and P3-1.5mg/Kg) exhibiting appearance of pyknotic nuclei (PN), fatty infiltrations (FD), inflammatory leukocyte infiltrations (LI) and activated Kupffer cells (KC), dilatation in blood sinusoids (DBS) and cytoplasmic vacuoles (VC).

\section{Discussion}

Our results have shown that following oral administration of phorate, there was a significant induction of hepatic P450 and lipid peroxidation. These results indicate that phorate probably acts as inducer of P450 isoenzyme. The augmented levels of cytochrome $\mathrm{P} 450$ would lead to high rates of radicals production, which, in turn, would favour increased rates of lipid peroxidation evidenced by the elevated MDA level. The increased activity of SOD and catalase may be due to the adaptive response to the generated free radicals (Koner et al., 1998) indicating the failure of the total antioxidant defence mechanism to protect the tissues from oxidative damage caused by pesticide, through free radicals . Bagchi et al. (1995) have shown that different classes of pesticides induce the production of reactive oxygen species (ROS) and oxidative tissue damage. Other reports indicate that the enzyme activities associated with antioxidant defence mechanisms are altered by insecticides both in vivo and in vitro (Patel and Chakrabarti 1982; Gultekin et al., 2000b, 2001; Oncu et al., 2002). The decreased activities of CAT, SOD and GSH level and increased MDA level in liver as well as increased serum AST, ALT, ALP activities suggest that phorate could causes hepatic damage.

Phorate could also cause a significant decrease in GSH activity. Blum and Fridovich (1985) showed that superoxide radicals could inhibit the activity of GSH. Increased ROS might be the reason for the inhibition of GSH activity. It has also been reported that various OPIs caused a decrease in GSH activity (Naqvi and Hasan, 1992; Yamano and Morita, 1992). Also it was shown that the administration of a pesticide mixture reduced the activities of GSH-Px in rat liver; the reduced enzyme activity was highly dependent on the increased dose of pesticide mixture (Lodowici et al., 1994). Finally, apart from biochemical alterations, there were clear ballooning of hepatocytes with focal inflammation. The histological changes suggest increasing frequency of hepatocellular damage with increasing dose of phorate in the animals.

\section{Conclusion}

In conclusion, the present findings indicate that the toxicity of phorate proceeds via derangement of the cellular oxidative status as evidenced by increased levels of lipid peroxidation, activity of transaminases and an altered antioxidant status, i.e., decreased GSH and lowered activities of CAT and SOD. Histopathological studies also confirm the hepatocellular damage. It could be concluded that phorate treatment induces oxidative stress mediated hepatotoxicity. 


\section{References}

[1]. ACG99 American Conference of Governmental Industrial Hygienists (ACGIH). Phorate. In: TLVs® and other occupational exposure values - 1999. [CD-ROM]. Cincinnati OH, USA: ACGIH®, Inc, 1999.

[2]. Almedia, M.G., Fanini, F., Davino, S., Aznar, A.E., Koch, O.R., Barros, S.B.M., 1997. Pro and antioxidant parameters in rat liver after short-term exposure to hexachlorobenzene. Hum. Exp. Toxicol. 16, 257-261.

[3]. Banerjee, B.D., Seth, V., Bhattacharya, A., Pasha, S.T., Chakraborty A.K., 1999. Biochemical effects of pesticides on lipid peroxidation and free radicals scavengers. Toxicol. Lett. 107, 33- 77.

[4]. Bachowski, S., Kolaja, K.L., Xu, Y., Ketcham, C.A., Stevenson, D.E., Walborg, E.F., Klaunig, J.E., 1997. Role of oxidative stress in the mechanism of dieldrin's hepatotoxicity. Annals of Clinical and Laboratory Science 27 (3), 196-209.

[5]. Bagchi, D., Bagchi, M., Hassoun, E.A., Stohs, S.J., 1995. In vitro and in vivo generation of reactive oxygen species, DNA damage and lactate dehydrogenase leakage by selected pesticides. Toxicology 104, 129-140.

[6]. Blum, J., Fridovich, I., 1985. Inactivation of glutathione peroxidase by superoxide radical. Archives of Biochemistry and Biophysics 240 (2), 500-508.

[7]. Chantelli-Forti, G., Paolini, M. and Hrelia, P. 1993. Multiple end point procedure to evaluate risk from pesticides. Environ Health Perspect. 101: 15-20.]

[8]. Chaudhuri, K., Selvaraj,S. and Pal, A.K. 1999.Studies on the genotoxicity of endosulfan in bacterial systems. Mutat. Res. 439: 63-67.

[9]. Dipanker, D., Tapas, M., 2000. Study of quinalphos formulation induced damage of testicular tissues and antioxidant. J. Appl. Toxicol. 20, 197-204.

[10]. Datta, J., Gupta (Dasgupta), J., Sarkar, A., Sengupta, D., 1992. Effects of organophosphorus insecticide phosphomidon on antioxidant defence components of human erythrocyte and plasma. Indian Journal of Experimental Biology 30, 65-67.

[11]. Escobar, J.A., Rubio, M.A., Lissi, E.A., 1996. SOD and catalase inactivation by singlet oxygen and peroxyl radicals. Free Radical Biology and Medicine 20, 285-290.

[12]. Ga191 Gallo MA, Lawryk NJ. Phorate. In: Hayes WJ Jr, Laws ER Jr, eds. Classes of pesticides.

[13]. San Diego CA, USA: Academic Press, Inc, 1991: 1055-7 (Handbook of pesticide toxicology; Vol 2, Sect 16.7.4).

[14]. Gultekin, F., Ozturk, M., Akdogan, M., 2000b. The effect of organophosphate insecticide chlorpyrifos ethyl on lipid peroxidation and antioxidant enzymes (in vitro). Archives of Toxicology 74, 533-538.

[15]. Gupta (Dasgupta), J., Datta, J., Sarkar, A., Sengupta, D., 1992. Effect of malathion on antioxidant defence system in human fetus - an in vitro study. Indian Journal of Experimental Biology 30, 352- 354.

[16]. Gultekin, F., Delibas, N., Yasar, S., Kilinc, I., 2001. In vivo changes in antioxidant systems and protective role of melatonin and a combination of vitamin $\mathrm{C}$ and Vitamin $\mathrm{E}$ on oxidative damage in erythrocytes induced by chlorpyrifos-ethyl in rats. Archives of Toxicology 75, 88-96.

[17]. Gultekin, F., Delibas, N., Arican, A., Sutcu, R., Kilinc, I., Kaptanagasi, M., 2000a. Investigation of the relation between LD50 and lipoperoxidative effect of certain agrochemical. Biomedical Research 11 (1), 67-71.

[18]. King, E.J., Armstrong, A.R. (1934) In vitro determination of alkaline phosphatases $C$ anad. Med. Ass. J. 31: 376-9

[19]. Kono, Y., Fridovich, I., 1982. Superoxide radical inhibits catalase. Journal of Biological Chemistry 257, 5751-5754.

[20]. Lemaire, P., Mathews, A., Forlin, L., Livingstone, D.R., 1994. Stimulation of oxyradical production of hepatic microsomes of flounder (Platichthys flesus) and perch (Perca fluviatilis) by model and pollutant xenobiotics. Arch. Environ. Contam. Toxicol. $26,191-200$

[21]. Lodowici, M., Aiolli, S., Monserrat, C., Dolara, P., Medica, A., Di Smlicio, P., 1994. Effect of a mixture of 15 commonly used pesticides on DNA levels of 8-hydroxy- 2- deoxyguanosine and xenobiotic metabolizing enzymes in rat liver. Journal of Environmental Pathology, Toxicology and Oncology 13 (3), 163-168.

[22]. Lowry, O.H., Rosebrough, N.J., Farr, A.L., Randall, R.J., 1951. Proteinmeasurement with Folin's phenol reagent. J. Biol. Chem 193, 265-275.

[23]. Maiti, P.K., Kar, A., 1997. Dimethoate inhibits extrathyroidal 50-monodeiodination of thyroxin to 3,30,5-triiodothyronine in mice: the possible involvement of the lipid peroxidative process. Toxicology Letters 91, 1-6.

[24]. Naqvi, S.M., Hasan, M., 1992. Acetylhomocystein thiolactone protection against phosphamidon-induced alteration of regional superoxide dismutase activity in the central nervous system and its correlation with altered lipid peroxidation. Indian Journal of Experimental Biology 30, 850-852.

[25]. Ohkawa, H., Ohishi, N., Yagi, K., 1979. Assay of lipid peroxidation on animal tissues by thio barbituric acid reaction. Anal. Biochem. 95, 351-358.

[26]. Omura, T., Sato, R., 1964. The carbon monoxide-binding pigment of liver microsomes II, solubilization, purification and properties. J. Biol. Chem. 239, 2370-2378.

[27]. Oncu, M., Gultekin, F., Karaoz, E., Altuntas, I., Delibas, N., 2002. Nephrotoxicity in rats induced by chlorpryfos-ethyl and ameliorating effects of antioxidants. Human and Experimental Toxicology 21 (4),223-230.

[28]. Patel, P.G., Chakrabarti, C.H., 1982. Changes in the activity of some hepatic enzymes during rganophosphorus insecticideacephate (orthene) treatment in albino rats. Indian Journal of Physiology and Pharmacology 26 (4), 311-316.

[29]. Reitman S, Frankel S. A colorimetric method for the determination of glutamic oxaloacetic and glutamic pyruvic transaminase. Am J Clin Pathol 1957;28:56-63.

[30]. Samanta, L., Chainy, G.B., 1995. Hexachlorocychohexane-induced changes in lipid peroxidation,superoxide dismutase and catalase activities and glutathione content in chick liver. Ind. J. Exp.Biol. 33, 131-133.

[31]. Tom97 Tomlin CDS, ed. In: The Pesticide Manual. A world compendium. 11th ed. Farnham (Surrey), UK: British Crop Protection Council, 1997.

[32]. Tietz, N.W. (1986) Textbook of clinical chemistry, Co. London: Saunder, W. B.; pp: 960-962

[33]. Vandana, S., Poovolla, V., 1999. Role of reactive oxygen metabolites in organophosphate bidrine-induced renal tubular cytotoxicity $10,1746-5172$.

[34]. Yamano, T., Morita, S., 1992. Hepatotoxicity of trichlorfon and dichlorvos in isolated rat hepatocytes. Toxicology 76, 69-77. 